\title{
Enantiodivergent non-linear effects in asymmetric catalysis
}

Yannick Geiger, Thierry Achard, Aline Maisse-François and Stéphane Bellemin-Laponnaz.*

\begin{abstract}
In this paper, we theoretically discuss the enantiodivergent product formation in asymmetric catalysis, a process in which the sign of the overall product enantiomer switches upon a change of catalyst concentration. The presented model is based on a catalytic system that consists of both discrete and dimeric aggregated metal complexes, in competition and in equilibrium. These concepts were then expanded to a non-enantiopure catalyst, giving rise to enantiodivergent non-linear effects - a special case of a hyperpositive non-linear effects where the product enantiomer's sign switches upon a change of the catalyst enantiomeric excess. Different cases are considered allowing a discussion of the influence of the parameters governing both models. Finally, we present experimental results that support the enantiodivergence while varying the concentration of enantiopure catalyst or while varying the enantiomeric excess of the catalyst, using chiral $\mathrm{N}$-methylephedrine as a ligand for the enantioselective addition of dimethylzinc to benzaldehyde.
\end{abstract}

\section{Introduction}

Non-linear effects (NLEs) in asymmetric catalysis refer to cases in which the enantiomeric excess of the product does not scale linearly with the enantiomeric excess of the catalyst. ${ }^{1}$ The first examples and models of such behavioural differences between scalemic and enantiomerically pure catalysts have been established by Kagan in $1986 .{ }^{2}$ Since then NLEs are considered as ubiquitous phenomena that provide additional information regarding the aggregation state of the catalyst or the formation of multiligand species. ${ }^{2-5}$ Not only being indicative of the catalytic system, NLEs also give clues to discussions on the origin of molecular homochirality in biology which is related to the origin of life. ${ }^{6}$

Several models for NLEs have been described and discussed in the literature, all of them being the results of interactions between the enantiomers of the chiral catalyst thus generating diastereomeric perturbations of the entire system. A positive non-linear effect (i.e.

Institut de Physique et Chimie des Matériaux de Strasbourg (IPCMS), Université de Strasbourg-CNRS UMR 7504, France. *e-mail: bellemin@unistra.fr 
asymmetric amplification) is essentially generated by the presence of a reservoir of racemic ideally catalytically inactive hetero-aggregate (meso). ${ }^{7-9}$ Amongst these models, Kagan established a theoretical case on where an unprecedented phenomenon could occur - that is the chiral catalyst will be much more efficient when partially resolved than when enantiomerically pure. We recently have observed such a case experimentally (known as hyperpositive NLE) and proposed a model that accounts for this observation. ${ }^{10}$ Our studies on the system pointed towards a two-component catalysis where mononuclear as well as dinuclear catalysts are in equilibrium and in competition. Through the precipitation of a heterochiral aggregate, varying the ligand ee lead to a change of catalyst concentration and, therefore, to a shift in the monomer-dimer equilibrium, thus favouring the more enantioselective monomeric catalyst at low ligand ee.

Here, we wish to illustrate with a mathematical model to offer a better understanding of such a two-component catalytic system. First, we calculated the enantiomeric excess of the product $\left(\mathrm{ee}_{\mathrm{P}}\right)$ as a function of the overall catalyst's concentration ([Cattot]) of an enantiopure catalytic system. Then we extended the model for a scalemic mixture of the catalyst and calculated $e_{P}$ as a function of the enantiomeric excess of the ligand (ee $\mathrm{L}_{\mathrm{L}}$. Numerical analyses of certain specific cases demonstrate that the sign of the enantiomeric product could change by varying [Cattot] or $e_{L}$, a conclusion that stimulated further experiments and which lead to the discovery of an enantiodivergent non-linear effect in asymmetric catalysis.

\section{Results and discussion}

\section{Enantiopure system: product ee as function of catalyst concentration}

$$
\begin{aligned}
& \mathrm{M}+\mathrm{L}_{\mathrm{R}} \longrightarrow[\mathrm{R}]+[\mathrm{RR}] \\
& \begin{array}{l|l}
\mathrm{k}_{1} & \\
\mathrm{ee}_{1} & \quad \mathrm{k}_{2} \\
\mathrm{ee}_{2} & \downarrow
\end{array}
\end{aligned}
$$

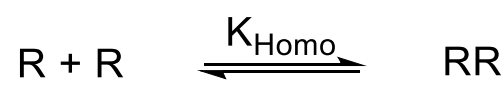

$$
\begin{aligned}
& \text { Product }\left(e e_{\mathrm{P}}\right) \\
& \mathrm{K}_{\text {Homo }}=\frac{[\mathrm{RR}]}{[\mathrm{R}]^{2}} \\
& {\left[\mathrm{Cat}_{\mathrm{tot}}\right]=[\mathrm{R}]+2[\mathrm{RR}]}
\end{aligned}
$$

Figure 1. Schematic representation of Model I, which consists of a monomeric and a dimeric enantiopure catalyst that both operate at a steady state. The catalysts are issued from the reaction of a metal salt (M) with a chiral, enantiopure ligand $\left(L_{R}\right)$. 
In order to describe the enantiomeric excess of the product $\left(\mathrm{ee}_{\mathrm{P}}\right)$ as a function of [Cat ${ }_{\text {tot }}$ ], we defined a system (Model $\mathrm{I}$ ) in which both monomeric and dimeric homochiral complexes ( $\mathrm{R}$ and $R R$, respectively) catalyse the reaction at different rates $\left(k_{1}\right.$ and $\left.k_{2}\right)$ and with different enantioselectivities (ee $e_{1}$ and $e_{2}$ ), as shown in Figure 1. The $[R R] /[R]$-ratio is considered to be constant and dependent only on the homochiral dimerization constant $\mathrm{K}_{\text {Homo }}$ (this constant is different to the one defined by Noyori which is a dissociation constant). ${ }^{9,11}$ This is somewhat related to Kagan's static $M L_{n}$ models, which stands in contrast to the dynamic (and much more complex) Noyori model. By combining the set of equations displayed in Figure 1, it was possible to obtain equations ( 1 ) and (2) which relate ee $\mathrm{P}$ to the parameters $\mathrm{k}_{1} / \mathrm{k}_{2}, \mathrm{ee}_{1}, \mathrm{ee}_{2}, \mathrm{~K}_{\mathrm{Homo}}$ and [Cattot] (full details for the calculations are displayed in the Supplementary Methods).

$$
\begin{gathered}
e e_{P}=\frac{\frac{k_{1}}{k_{2}} e e_{1}+\gamma e e_{2}}{\frac{k_{1}}{k_{2}}+\gamma} \\
\gamma=\frac{\sqrt{1+8 K_{\text {Homo }}[\mathrm{Cat} t o t]}-1}{4}
\end{gathered}
$$

If we suppose that $e_{1}>e_{2}$, the overall enantiomeric excess of the product ee $e_{P}$ will increase with the decrease of the catalyst concentration [Cat ${ }_{\text {tot }}$ ] as the equilibrium will be shifted to the more enantioselective monomeric R-catalyst. Such feature can account for hyperpositive non-linear effects, as previously observed by us in the case of the NBE-catalysed system. ${ }^{10}$

An interesting case could be encountered here: "less enantioselective" may also mean that the dimeric RR-catalyst could give the other enantiomer as product: $e_{2}$ then takes a negative value. In such a system, a simple change of the overall catalyst concentration [Cat ${ }_{\text {tot] }}$ - and, therefore, of the $[R R] /[R]$-ratio- might be sufficient to switch the sign of the product enantiomer. To shed light on this possibility, we computed various curves obtained from equations (1) and (2) for several cases (Figure 2). Figure 2a displays the evolution of ee for different values of ee $e_{2}$ with fixed values of $K_{\text {Homo, }} k_{1}$ and $k_{2} ; k_{1} / k_{2}$ was set to 1 and $K_{\text {Homo }}$ to 33 , which corresponds to DAIB's $K_{H_{0 m o}}$-value. ${ }^{9,11}$ The three graphs in Figure 2 show that ee ${ }_{P}$ can become negative when $e_{2}<0$ and when [Cattot] is sufficiently high; the lower $e_{2}$, the lower the value at which the plot crosses the [Cat ${ }_{\text {tot }}$-axis. In the same way, the more the reaction is dominated by the dimeric catalyst - that is, with a high $[R R] /[R]$-ratio (i.e. high $\mathrm{K}_{\text {Homo, }}$ Figure $2 b)$ and/or a higher rate of the dimer-catalysed reaction (i.e. low $k_{1} / k_{2}$, Figure $2 c$ ) - the more $e_{P}$ is negative and the more the plot becomes hyperbolic, thus requiring very low [Cat ${ }_{t o t}$ to generate a positive ee $e_{p}$. The value at which the curve crosses the [Cat ${ }_{\text {tot }}$-axis (i.e. $e_{P}=0$, 
labelled as $\left[\mathrm{Cat}_{\text {tot }}{ }^{0}\right.$ ) corresponds to an overall catalytic system where $\mathrm{R}$ and $\mathrm{RR}$ catalysts compensate each other to yield an overall racemic product - even if both give independently enantiopure products.
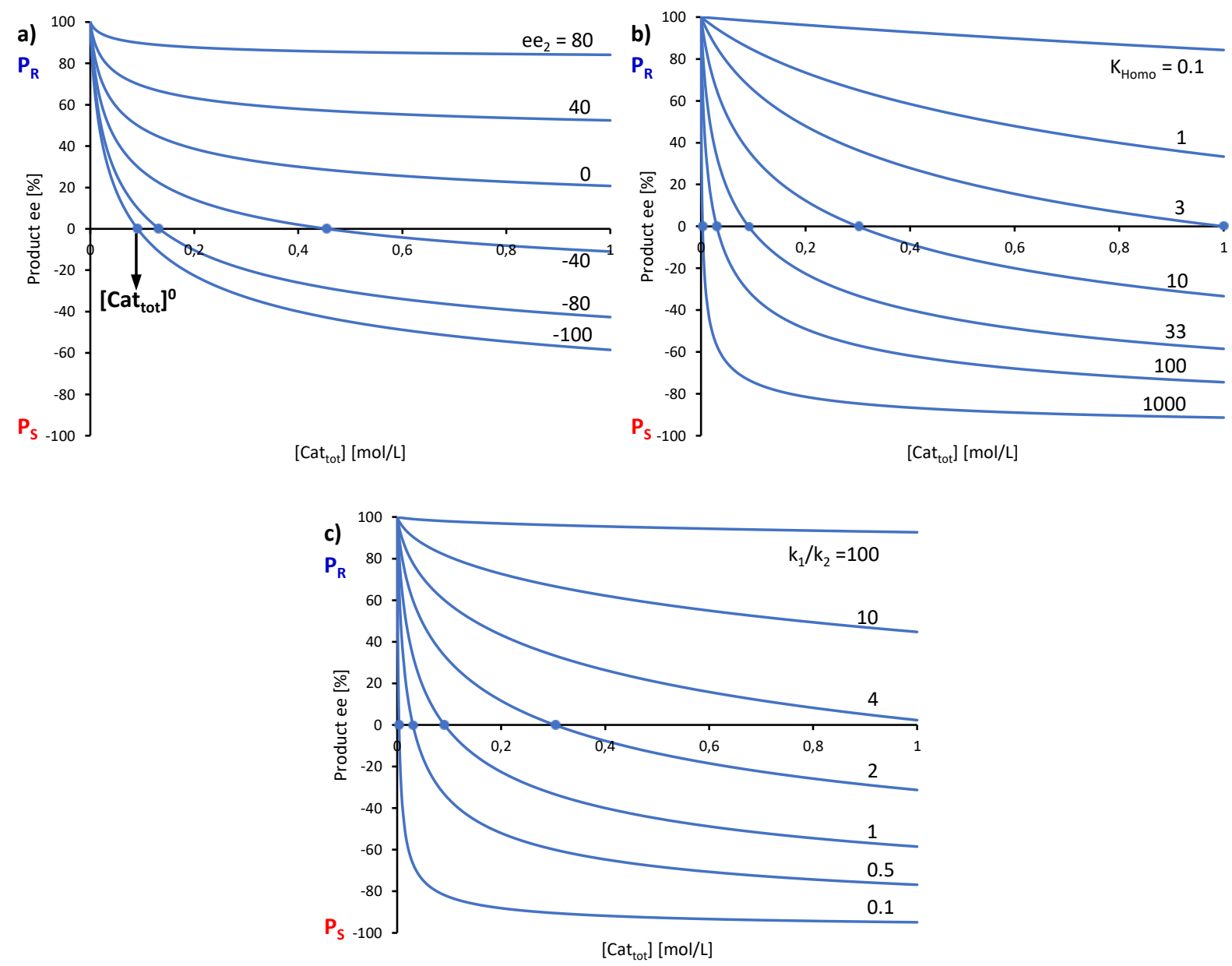

Figure 2. Simulation of the relationship between eep and [Cattot] according to equations (1) and (2). The basic set of parameters is ee $1=100, e_{2}=-100, K_{\text {Homo }}=33$ and $k_{1} / k_{2}=1$. Each panel shows curves where one of the parameters has been varied: a) ee 2 , b) $\left.K_{\text {Homo }}, c\right) k_{1} / k_{2}$. The product ee is defined as $\left(\mathrm{P}_{\mathrm{R}}-\mathrm{PS}_{\mathrm{S}}\right) /\left(\mathrm{P}_{\mathrm{R}}+\mathrm{P}_{\mathrm{S}}\right)$.

A catalytic system generating either one or the other product enantiomer by changing parameters other than the configuration of the catalyst is called enantiodivergent. Over the last two decades, several examples have been reported in which slight changes of the catalyst (substituent, metal, counter-ion, etc.) or of the reaction conditions (solvent, temperature, additive, ligand-to-metal ratio) have inverted the stereochemistry of the product. ${ }^{12-15}$ However, the catalyst concentration had never been discussed so far in this context. We are aware of one case where the concentration of the catalyst had some influence on the stereochemical 
outcome of an enantioselective reaction, however the most influencing parameter was the ligand-to-metal ratio. ${ }^{16}$

\section{Scalemic system: product ee as function of ligand ee}

The outcome from Model I provided the context to consider the possibility of obtaining both product enantiomers also by varying the ligand ee $\left(e_{\mathrm{L}}\right)$ without the need to switch the sign of the catalyst - in analogy to the common terminology, this would be called an enantiodivergent non-linear effect. The catalyst distribution is generally influenced by $e_{\llcorner}$when a NLE is present, thus it is conceivable that a change in ee $e_{L}$ induces changes in the ratio between both catalysts that give opposite product enantiomer, as in the discussion on Model I. Du and co-workers reported an example for an enantiodivergent NLE, where a Zn-BINOLcatalyst yielded the opposite product enantiomer in a hetero-Diels-Alder reaction when used in low ee . $^{17,18}$ However, the origins of the enantiodivergent behaviour have not been studied or discussed further. In Model II, we made an extension of Model I to non-enantiopure catalysts and studied some numerical cases (Figure 3).

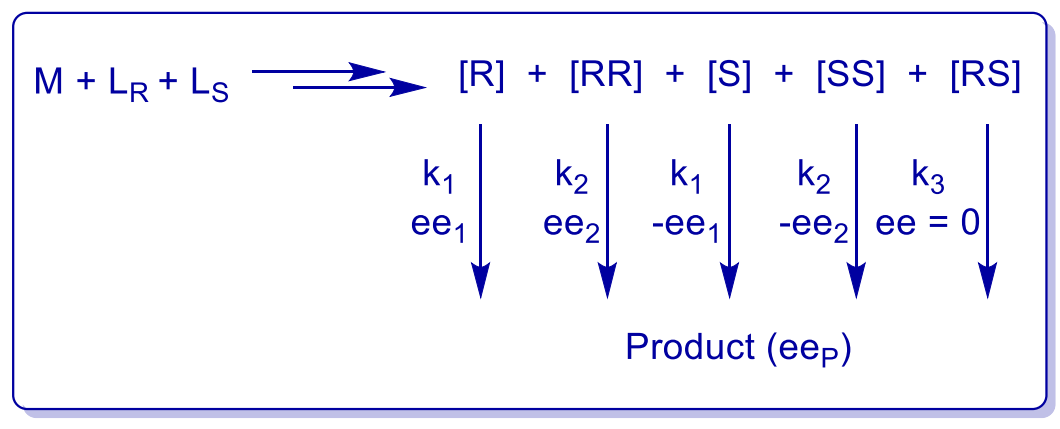

$$
\left[\text { Cat }_{\text {tot }}\right]=[R]+2[R R]+[S]+2[S S]+2[R S]
$$

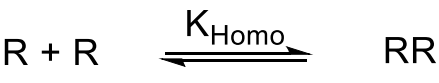

$$
\begin{aligned}
& \mathrm{S}+\mathrm{S} \stackrel{\mathrm{K}_{\text {Homo }}}{=} \mathrm{SS} \\
& \mathrm{K}_{\mathrm{Homo}}=\frac{[\mathrm{RR}]}{[\mathrm{R}]^{2}}=\frac{[\mathrm{SS}]}{[\mathrm{S}]^{2}} \\
& \mathrm{R}+\mathrm{S} \stackrel{\mathrm{K}_{\text {Hetero }}}{=} \mathrm{RS} \\
& \mathrm{K}_{\text {Hetero }}=\frac{[\mathrm{RS}]}{[\mathrm{R}][\mathrm{S}]}
\end{aligned}
$$

Figure 3. Schematic representation of Model II, which consists of monomeric and both homo- and heterochiral dimeric catalysts that all operate at a steady state. The catalysts are issued from the reaction of a metal salt $(M)$ with a mixture of both ligand enantiomers $\left(L_{R}\right.$ and $\left.L_{s}\right)$.

Figure 3 shows the catalytic system of Model II. While the prerequisites are identical to Model I, the difference is in the enantiomeric purity of the total catalyst which may be comprised between $0-100 \%$ ee. This implies the presence of the catalytic species S and SS into the model (which are linked through $\mathrm{K}_{\text {Homo }}$ as their enantiomeric counterparts) as well as the heterochiral dimer $\mathrm{RS}$, which is related to $\mathrm{R}$ and $\mathrm{S}$ through the dimerization constant $\mathrm{K}_{\text {Hetero }}$ and may generate racemic products with a rate constant $k_{3}$. To build the mathematical expressions, we 
used the approach used by Noyori for the DAIB-model which consists in introducing $\alpha=[R]+$ $[S]$ and $\beta=[R][S]$ to simplify the equations. $e_{P}$ and $e_{L}$ are then given by equations (3) and (4). Since $\beta$ is itself a function of $\alpha$ [cf. equation (5)], ee $e_{P}$ and $e_{L}$ are linked through $\alpha$ and depend only on the parameters $\mathrm{K}_{\text {Homo }}, \mathrm{K}_{\text {Hetero }}, \mathrm{k}_{1}, \mathrm{k}_{2}, \mathrm{k}_{3}$, ee ${ }_{1}$, ee $\mathrm{e}_{2}$ and [Cattot]. After defining these parameters, ee $v s$ ee $e_{\mathrm{L}}$-datasets can be obtained by choosing appropriate values for $\alpha$. The details of the calculations, as well as the general expressions for the upper and lower limits of $\alpha$, can be found in the Supplementary Methods.

$$
\begin{gathered}
e e_{P}=\frac{\sqrt{\alpha^{2}-4 \beta}\left(k_{1} e e_{1}+\alpha K_{\text {Homo }} k_{2} e e_{2}\right)}{\alpha k_{1}+\left(\alpha^{2}-2 \beta\right) K_{\text {Homo }} k_{2}+\beta K_{\text {Hetero }} k_{3}} \\
e e_{L}=\frac{\sqrt{\alpha^{2}-4 \beta}\left(1+2 \alpha K_{\text {Homo }}\right)}{\left[\text { Cat }_{\text {tot }}\right]} \\
\beta=\frac{\left(\alpha+2 K_{\text {Homo }} \alpha^{2}-\left[\text { Cat }_{\text {tot }}\right]\right)}{4 K_{\text {Homo }}-2 K_{\text {Hetero }}}
\end{gathered}
$$

Because of the large number of parameters governing Model II, one can give a multitude of different curves. For this study, we wish to focus on the cases where the NLE is hyperpositive and potentially enantiodivergent - that is with $\mathrm{K}_{\text {Hetero }}>2 \mathrm{~K}_{\text {Homo }}$ which is, as in the Noyori model, a necessary condition to obtain (+)-NLEs) and $e e_{1}>e_{2} .^{9}$ Figure 4 shows several cases computed from to Model II; to simplify the discussion we have set $\mathrm{k}_{3}=0$ in all simulations except in case of Figure $4 \mathrm{f}$.

Influence of $\mathbf{K}_{\text {Homo, }}$ [Cat ${ }_{\text {tot }}$ ], $\mathbf{k}_{\mathbf{1}} / \mathbf{k}_{\mathbf{2}}$ and $\mathbf{e e}_{\mathbf{1 / 2}}$. A non-linear effect is hyperpositive as long as its highest product ee (labelled as $e_{P}{ }^{\max }$ ) is higher than the $e_{P}$ for the enantiopure ligand $\left(e e_{P}^{100}\right)$. In Model II, ee ${ }^{100}$ will be strongly dependent on $\mathrm{K}_{\text {Homo }}$, [Cattot] and $\mathrm{k}_{1} / \mathrm{k}_{2}$ (Figure $4 \mathrm{a}-\mathrm{c}$ ): the higher

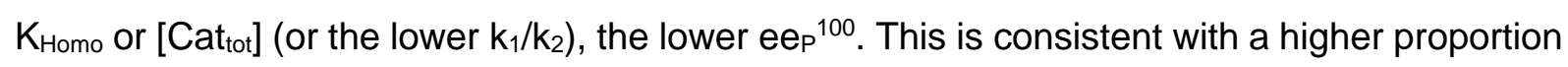
and a higher activity of the low ee -yielding RR-catalyst over its monomeric counterpart.

In all panels, we selected conditions in which ee $^{100}$ could be negative and where the NLE curve crosses the ee $e_{L}$-axis, making it an enantiodivergent NLE. The lower ee $_{P}{ }^{100}$, the lower the crossing point at which $e_{P}=0\left(e_{L}{ }^{0}\right)$. At this point, the outcomes of all catalysts

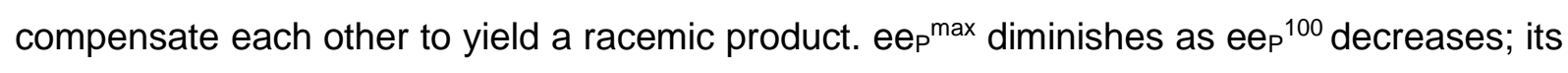
position on the ee ${ }_{L}$-axis (ee ${ }_{L}{ }^{m a x}$ ) is only slightly affected by $\mathrm{K}_{\text {Homo }}$ and in a somewhat greater extend by [Cattot] and $k_{1} / k_{2}$. This is seen nicely if, for a given set of parameters, $K_{\text {Homo }}$ is multiplied and [Cattot] divided by the same value: $e_{P}{ }^{100}$ stays unchanged but ee $_{P}{ }^{\text {max }}$ and $e e^{\text {max }}$ do not (cf. Supplementary Figure 1). Figure $4 d$ shows the impact of $e e_{1}$ and $e e_{2}$ on $e e^{100}$ : the 
lower $e_{2}$, the lower $e^{100}$. This is also true for $e^{1}$; however, if both $e_{1}$ and $e_{2}$ are negative, the enantiodivergent NLE curve becomes a classic (+)-NLE for the S-product ( $P_{S}$, Figure $4 d$, dashed curves); the same holds for the R-product $\left(P_{R}\right)$ if $e e_{1} \leq e_{2}$ and if both are positive. In a similar way, very high $\mathrm{K}_{\mathrm{Homo}_{0}} /\left[\right.$ [Cattot]- or very low $\mathrm{k}_{1} / \mathrm{k}_{2}$-values lead to apparent (+)-NLEs as ee $_{P}{ }^{\max }$ and ee $^{\text {max }}$ become exceedingly close to 0 . Lowering the absolute amount of both a positive $e_{1}$ and a negative $e_{2}$ leads to a compression of the spectra (cf. Supplementary Figure 2).

Influence of $\mathbf{K}_{\text {Hetero }}$ and $\mathbf{k}_{3}$. In contrast to the previously discussed parameters, an increase in $\mathrm{K}_{\text {Hetero }}$ (Figure $4 \mathrm{e}$ ) does not affect $\mathrm{ee}_{\mathrm{P}}{ }^{100}$ but has a great impact on the hyperpositive maximum, which is shifted to higher $e_{P}{ }^{\max }$ and lower ee $_{L}{ }^{\max }$ values. Consequently, the $e^{0}{ }^{0}$ is shifted to higher $e_{\llcorner}$values under the same conditions. However, this is only true if the meso dimer is catalytically inactive: RS performing racemic catalysis $\left(k_{3} \neq 0\right.$, Figure $\left.4 f\right)$ leads to the inverse effect, namely a compacting of the curve. The values of $e{ }_{P}^{100}$ and $e^{2}{ }^{0}{ }^{~ r e m a i n ~ u n c h a n g e d, ~}$ the latter being an isobestic point. The value of $e_{P}{ }^{\max }$ decreases significantly even at low $\mathrm{k}_{3^{-}}$ values as the concentration of $R S$ at low ee $e_{L}$ is particularly high. High $k_{3}$-values $\left(k_{3}>k_{1}, k_{2}\right)$ additionally lead to a contraction of the curve between $e^{\circ}{ }^{0}$ and $e e_{L}=100$ and push its

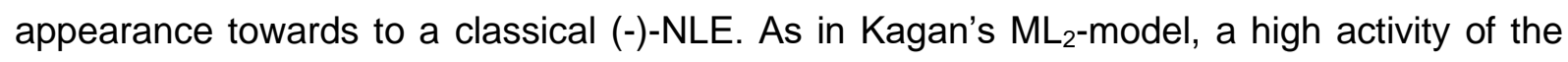
meso catalyst leads to asymmetric depletion. 

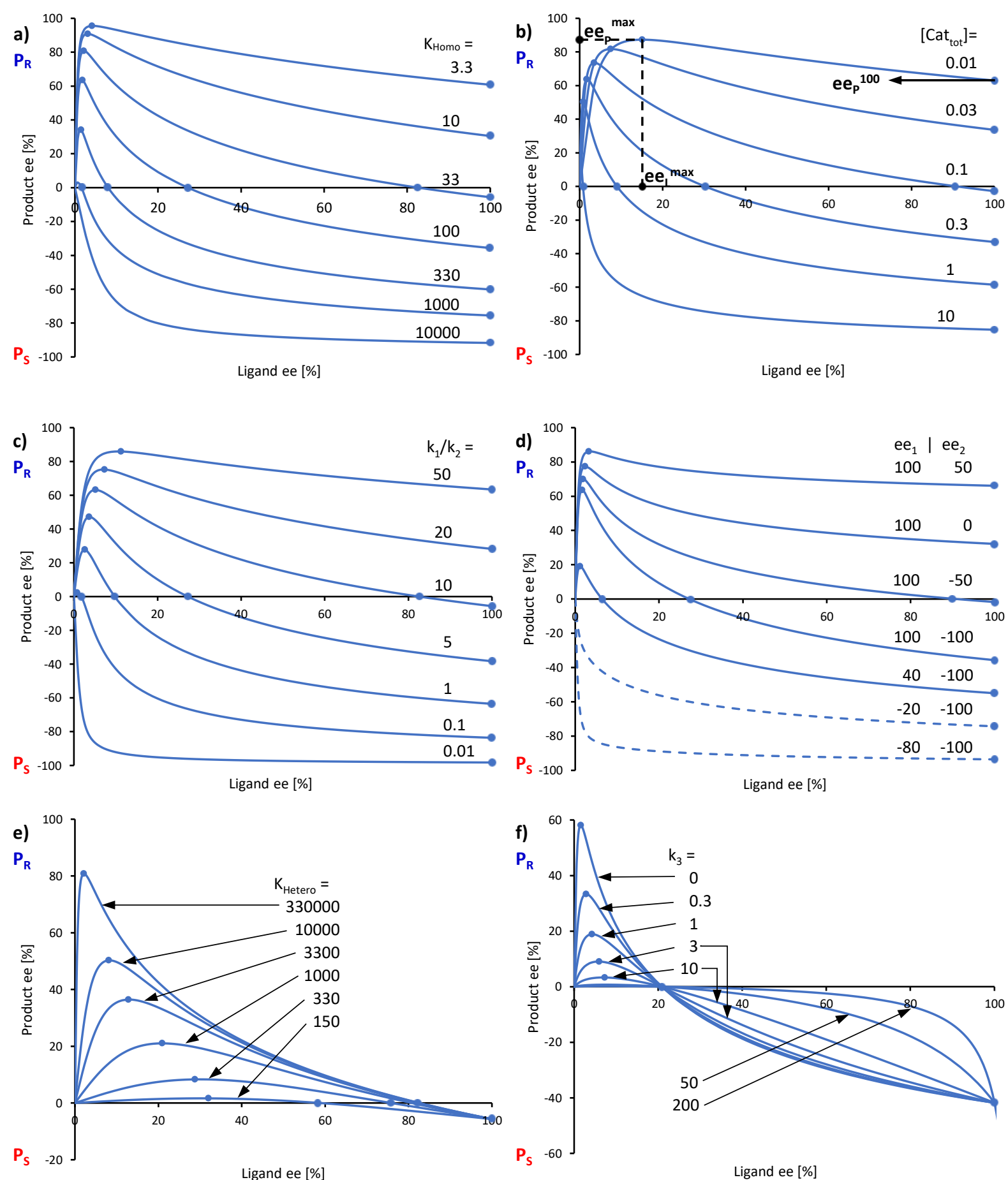

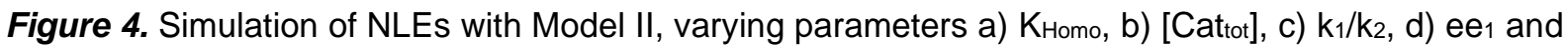
$\left.e e_{2}, e\right) K_{\text {Hetero }}$ and f) $k_{3}$. Fixed parameters: [Cattot] $=0.11, k_{1}=k_{2}=10, e e_{1}=100, e e_{2}=-100$ and $k_{3}=0$ in all curves except where the corresponding parameter is varied; $K_{\text {Homo }}=33$ (b, c, e), 100 (d) and 130 (f); $K_{\text {Hetero }}=330000$ (a, d, f), 100000 (b) and 33000 (c). The product ee is defined as $\left(P_{R}-P_{S}\right) /\left(P_{R}+P_{s}\right)$. Note: it is sufficient to consider only the $k_{1} / k_{2}$-ratio as long as $k_{3}=0$; otherwise $k_{1}$ and $k_{2}$ have to be treated as absolute values. 


\section{A case study: $N$-methylephedrine as chiral ligand}

A look into literature reveals that enantiodivergent catalytic systems where different ligand-to-metal-ratio give different product enantiomers are known. ${ }^{12-15}$ To the best of our knowledge, the first (and largely unnoticed) example was reported by Seebach and collaborators who found in his early works on TADDOL-Ti-catalyzed alkylations that a 2:1 ligand/metal ratio gives the opposite enantiomer than with a 1:1 ratio. ${ }^{19-21}$ Similar results were reported later by Porte, ${ }^{16}$ Danjo, ${ }^{22}$ Shao and Peng, ${ }^{23,24}$ Burguete ${ }^{25}$ and $\mathrm{Li}^{26}$ in various metalcatalyzed reactions. $2: 1$ and 1:1-complex ratio can be considered as analogues to the monomeric and dimeric complexes discussed so far (they even are equivalent in Kagan's $\mathrm{ML}_{2}$ model $^{7}$ ); therefore, the existence of catalytic systems with enantiodivergent NLEs outside of theory doesn't seem unrealistic. In light of our observations with NBE, ${ }^{10}$ this encouraged us to explore more deeply ephedrine-based ligands. To our delight, we found experimental evidence for such a enantiodivergent NLE using chiral (1R,2S)-(-)- $N$-methylephedrine [(-)-NME] as a ligand for the enantioselective addition of $\mathrm{ZnMe}_{2}$ to benzaldehyde (cf. reaction scheme in Figure 5). Interestingly, NME has been previously used in dialkylzinc additions using $\mathrm{ZnEt}_{2},{ }^{27-}$ ${ }^{30}$ with no NLE being observed. ${ }^{31}$ However, to the best of our knowledge, $\mathrm{ZnMe}_{2}$ has never been used as dialkylzinc reagent in NME-catalyzed reactions to this date.

Figure 5a shows the ee $\mathrm{P}$ vs. ee $\mathrm{L}$-plot of the (-)-NME-catalysed addition of $\mathrm{ZnMe}_{2}$ to 0.83 molar benzaldehyde at $0{ }^{\circ} \mathrm{C}$, which is a classic (-)-NLE with $P_{S}$ as the major enantiomer. The latter is surprising since the ligand's chiral configuration is the same as in (-)-NBE, which gives mainly the $R$-product. A screening of the catalyst loading using enantiopure (-)-NME (Figure 5b) revealed the catalytic system to be enantiodivergent: a catalytic charge of $20 \mathrm{~mol} \%(-)-$ NME yields product in $-11 \%$ ee, with $\mathrm{P}_{\mathrm{S}}$ being predominant. Lowering the catalyst loading progressively moves ee $\mathrm{P}_{\mathrm{P}}$ into the positive scale, giving $\mathrm{P}_{\mathrm{R}}$ in $18 \%$ ee at $2.5 \mathrm{~mol} \%$ and a [Cat $\left.{ }_{\text {tot }}\right]^{0}$ of ca. $9 \mathrm{~mol} \%$ (ca. $50 \mathrm{mM}$ ). By analogy with our previous investigations on NBE, we can extrapolate that this is a Model I-type system that contains two different catalysts: a $\mathrm{P}_{\mathrm{R}}$-giving monomer and a $\mathrm{Ps}_{\mathrm{s}}$-giving homochiral dimer which are both in equilibrium and in competition. Therefore, the (-)-NLE in Figure 5a follows Model II and the prevalence of the S-product comes from the catalytic system being dominated by the homochiral dimer. It should be noted that in

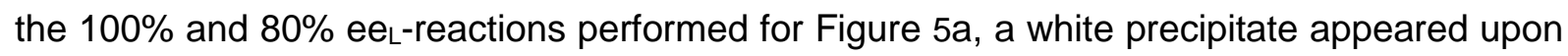
addition of $\mathrm{ZnMe}_{2}$ to (-)-NME which vanished over the course of the reaction; no precipitate was observed in the catalytic runs with lower $e_{L}$. This is most likely a homochiral precipitate which was also observed in a catalyst loading screening using the same conditions (cf. Supplementary Figure 3 for further discussion). In order to stay consistent with Model II, the catalyst loading screening in Figure $5 \mathrm{~b}$ and the following experimental studies were performed 
in more diluted conditions ( 0.56 instead of $0.83 \mathrm{M}$ benzaldehyde concentration) to avoid the formation of the precipitate.

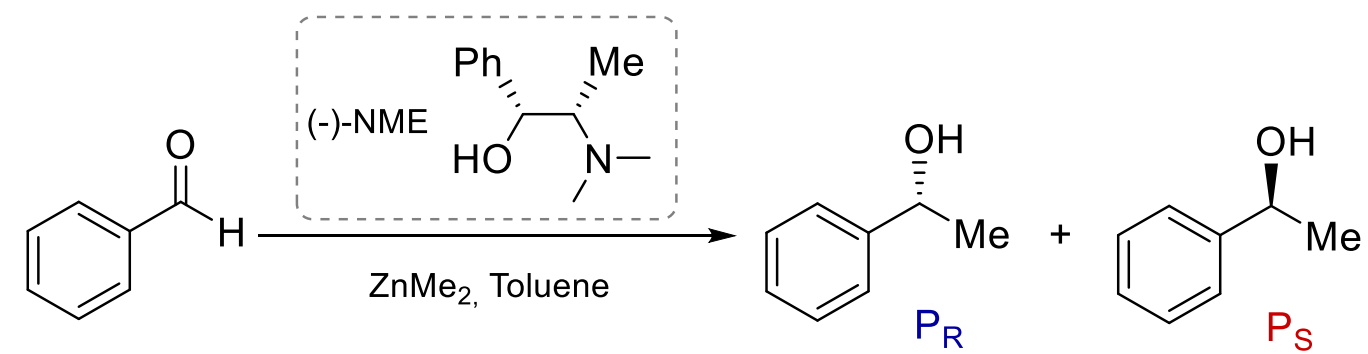

a) (-)-NME: $20 \mathrm{~mol} \%$, var. ee $\mathrm{ZnMe}_{2}: 1.2$ equivalents b) (-)-NME: $2.5-20 \mathrm{~mol} \%, 100 \%$ ee $\mathrm{ZnMe}_{2}: 1.025-1.2$ equivalents
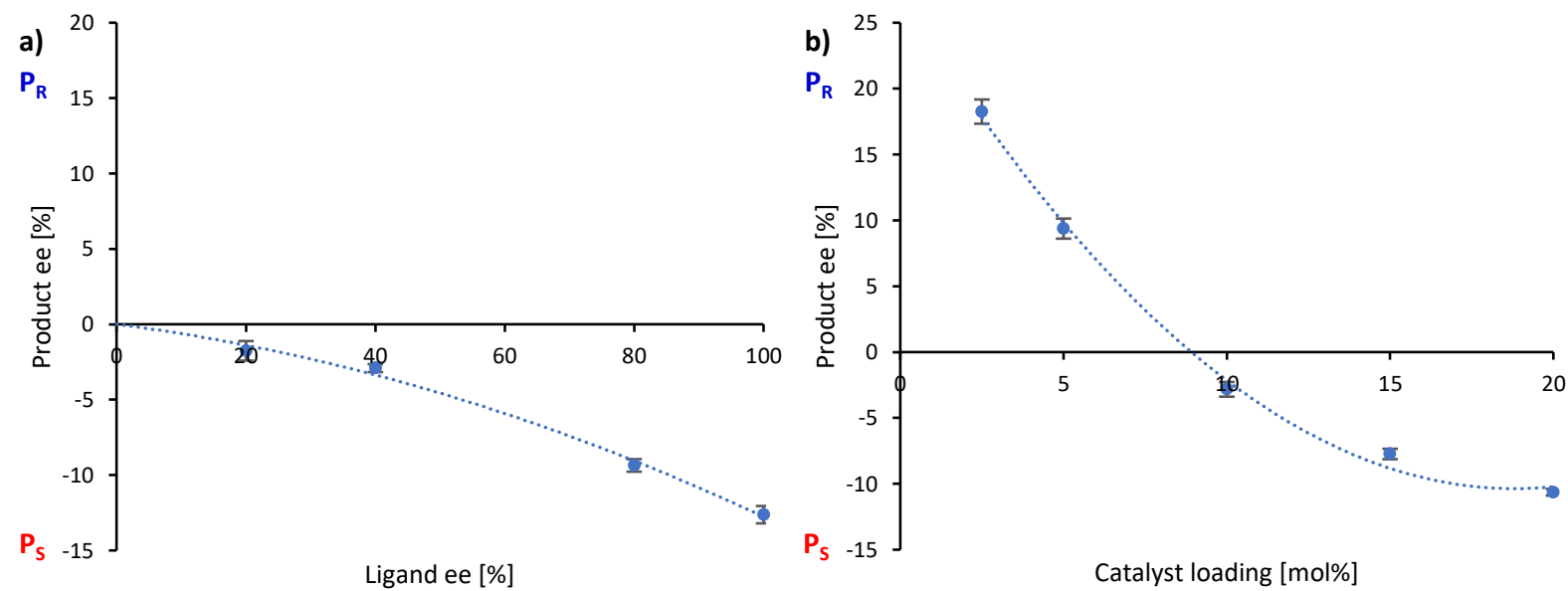

Figure 5. a) NLE-curve and b) eep vs catalyst loading-plot of the (-)-NME-catalyzed enantioselective addition of $\mathrm{ZnMe}_{2}$ to benzaldehyde $\left(0.83\right.$ and $0.56 \mathrm{M}$, respectively) at $0^{\circ} \mathrm{C}$. Each point is the mean of three independent experiments; the vertical bars depict standard deviations. The $2^{\text {nd }}$-order polynomial fits (dotted lines) are intended for illustration purposes only. The product ee is defined as $\left(\mathrm{P}_{\mathrm{R}}-\right.$ $\mathrm{PS}) /\left(\mathrm{P}_{\mathrm{R}}+\mathrm{PS}_{\mathrm{S}}\right.$.

We then wondered if a change in reaction temperature affect the NLE curve. Figure $6 \mathrm{a}$ shows a temperature screening of the reaction with the ligand having either $100 \%$ or $50 \%$ ee . At low temperature $\left(0^{\circ} \mathrm{C}\right)$ the product ee of the enantiopure ligand is well below the $e_{P}$ of the scalemic sample, both being negative. Increasing the temperature increases $e_{P}$ in both cases but not in the same manner: the difference between $100 \%$ and $50 \%$ ee L decreases

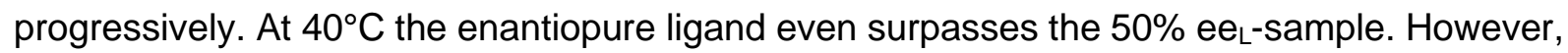
the most interesting point is the one at room temperature $\left(20-25^{\circ} \mathrm{C}\right)$ : here, $(-)-\mathrm{NME}$ in $50 \%$ ee gives positive eep-values while the enantiopure ligand stays negative - which is nothing but the requirement for an enantiodivergent non-linear effect. The full NLE curve at $20-25{ }^{\circ} \mathrm{C}$ (Figure $6 \mathrm{~b}$, blue dots) confirms this observation: the curve starts at $0 \%$ ee $\mathrm{e}_{\mathrm{L}}$ in the positive ee $\mathrm{P}^{-}$ 
range, reaches a maximum ( $\left(\mathrm{e}_{\mathrm{L}}{ }^{\max } \approx 50 \%, \mathrm{ee}_{\mathrm{P}}{ }^{\max } \approx 2 \%\right.$ ), then falls down to cross the ee ${ }_{\mathrm{L}}$-axis $\left(e^{\circ}{ }^{0} \approx 80 \%\right)$ and ends up in the negative part of the ee $e_{\mathrm{P}}$-scale. The use of $(+)$-NME (red squares) gives an appropriate mirror image of this curve.

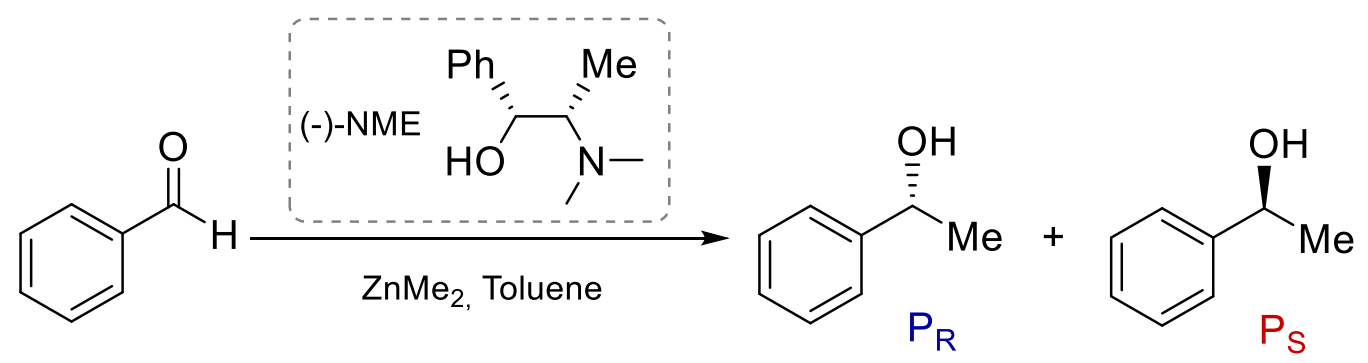

a) (-)-NME: $20 \mathrm{~mol} \%, 100 \%$ or $50 \%$ ee $\mathrm{ZnMe}_{2}: 1.2$ equivalents Var. temperatures b) (-) or (+)-NME: $20 \mathrm{~mol} \%$, var. ee $\mathrm{ZnMe}_{2}: 1.2$ equivalents Temperature: $20-25^{\circ} \mathrm{C}$
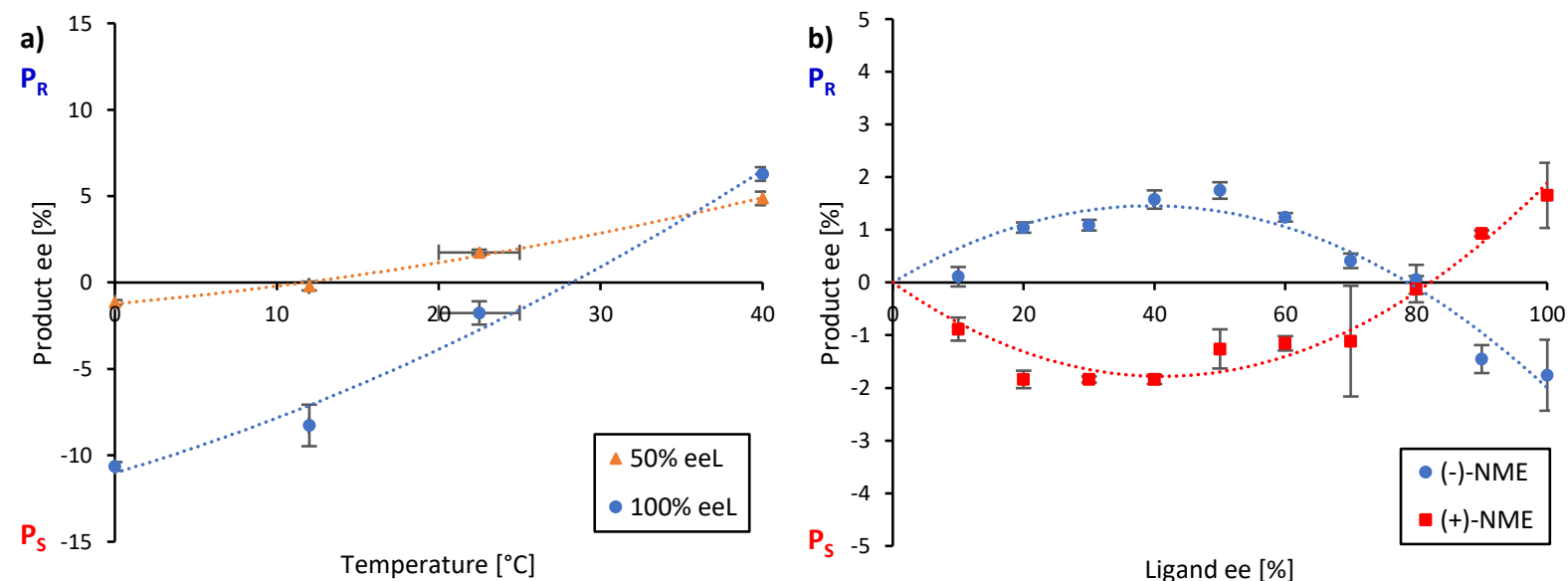

Ligand ee [\%]

Figure 6. a) eep as a function of the reaction temperature (blue dots: $100 \%$ eeL; orange triangles: $50 \%$ eeL) and b) NLE at room temperature of (-)-NME (blue dots) and (+)-NME (red squares) of the NMEcatalysed enantioselective addition of $\mathrm{ZnMe}_{2}$ to $0.56 \mathrm{M}$ benzaldehyde. Each point is the mean of three different experiments; the vertical bars depict standard deviations. The $2^{\text {nd }}$-order polynomial fits (dotted lines) are intended for illustration purposes only. The product ee is defined as $\left(\mathrm{P}_{\mathrm{R}}-\mathrm{PS}_{\mathrm{S}}\right) /\left(\mathrm{P}_{\mathrm{R}}+\mathrm{PS}_{\mathrm{S}}\right)$.

However, with the present data at hand, it is difficult to conclude on the thermodynamic parameters which govern the NME-catalyzed system. According to Model II, the overall small ee $e_{P}$ values with both (-)- and (+)-NME may originate from low $\mathrm{K}_{\text {Homo }}$ and $\mathrm{K}_{\text {Hetero, }}$ but this may not be true if $\mathrm{k}_{1} / \mathrm{k}_{2}$ deviates significantly from 1 or if $\mathrm{ee}_{2}$ has a small negative value. Considering the literature data about $\mathrm{ZnEt}_{2}$-additions, ${ }^{29} \mathrm{ee}_{1}$ probably exceeds $80 \%$ as the catalyst's enantioselectivity usually doesn't differ much between $\mathrm{ZnEt}_{2}$ and $\mathrm{ZnMe}_{2 .}{ }^{9} \mathrm{In}$ addition, Model I and Model II are probably not sufficiently adapted to the NME-catalysed system to allow 
complete and accurate quantitative studies. Dialkylzinc chemistry is known to be highly fluxional, the different alkylzinc intermediates depending on different dynamic equilibria and their concentrations fluctuating over the course of the reaction. ${ }^{9,10}$ However, Models I and II assume a static distribution of the different complexes. Further work is ongoing in our group to obtain more thermodynamic data from the NME- and NBE-catalysed systems and to develop more complex models which reflect the dynamic behaviour of alkylzinc species.

\section{Conclusion}

In conclusion, our recent discovery of a hyperpositive non-linear effect in an asymmetric catalysis allowed us to build models which accounts for the experimental results. The scope of the models has been assessed using different values and suggested the possibility of observing an interesting situation: the opportunity to find a catalytic system that generates both enantiomer products by varying the catalyst ee with no need to switch the sign of the catalyst. Based on this assumption, we have discovered an example of such an enantiodivergent nonlinear effect in asymmetric catalysis while studying the (-)-NME-catalysed enantioselective addition of $\mathrm{ZnMe}_{2}$ to benzaldehyde. Further work is in progress to get a deeper understanding of dual monomer/dimer-catalysed systems in enantioselective dialkylzinc additions, by both experimental and theoretical means.

\section{Methods}

General procedure for catalytic runs. In a $\mathrm{N}_{2}$-filled glovebox, $(1 R, 2 S)-N$-methylephedrine ${ }^{32}$ (17.9 mg, $20 \mathrm{~mol} \%, 100 \mu \mathrm{mol}$ ) and a magnetic stirring bar were placed in an oven-dried vial, which was then closed with a septum-containing screwcap. The vial was put out of the glovebox and dry toluene $(0.4 \mathrm{~mL})$ followed by a $1.2 \mathrm{M} \mathrm{ZnMe}_{2}$ solution in toluene $(0.5 \mathrm{~mL}, 1.2$ equiv, $600 \mu \mathrm{mol})$ were added via syringe; gas evolution occurred upon $\mathrm{ZnMe}_{2}$ addition. The mixture stirred for $10 \mathrm{~min}$, then benzaldehyde (51 $\mu \mathrm{L}, 1$ equiv, $500 \mu \mathrm{mol})$ was added via syringe. The solution stirred at room temperature for $48 \mathrm{~h}$, then was quenched carefully with 3 $\mathrm{M}$ aqueous $\mathrm{HCl}$ under vigorous stirring. The organic phase was then analysed by chiral stationary phase GC. 


\section{Data availability}

The complete mathematical treatment for equations (1)-(5) as well as additional computed curves, experimental results and details concerning the experimental methods are found in the Supplementary Information.

\section{References}

1. Kagan, H. B. \& Luukas, T. O. in Comprehensive Asymmetric Catalysis (eds. Jacobsen, E. N., Pfaltz, A. \& Yamamoto, H.) Ch. 4 (Springer-Verlag, Berlin, 1999).

2. Puchot, C. et al. Nonlinear effects in asymmetric synthesis. Examples in asymmetric oxidations and aldolization reactions. J. Am. Chem. Soc. 108, 2353-2357 (1986).

3. Kagan, H. B. Nonlinear Effects in Enantioselective Organometallic Catalysis. Oil \& Gas Science and Technology - Rev. IFP 62, 731-738 (2007).

4. Girard, C. \& Kagan, H. B. Nonlinear Effects in Asymmetric Synthesis and Stereoselective Reactions: Ten Years of Investigation. Angew. Chem. Int. Ed. 37, 2922-2959 (1998).

5. Satyanarayana, T., Abraham, S. \& Kagan, H. B. Nonlinear Effects in Asymmetric Catalysis. Angew. Chem. Int. Ed. 48, 456-494 (2009).

6. Blackmond, D. G. The origin of biological homochirality. Phil. Trans. R. Soc. B 366, 2878-2884 (2011).

7. Guillaneux, D., Zhao, S.-H., Samuel, O., Rainford, D. \& Kagan, H. B. Nonlinear effects in asymmetric catalysis. J. Am. Chem. Soc. 116, 9430-9439 (1994).

8. Kitamura, M., Okada, S., Suga, S. \& Noyori, R. Enantioselective addition of dialkylzincs to aldehydes promoted by chiral amino alcohols. Mechanism and nonlinear effect. J. Am. Chem. Soc. 111, 4028-4036 (1989).

9. Kitamura, M., Suga, S., Oka, H. \& Noyori, R. Quantitative Analysis of the Chiral Amplification in the Amino Alcohol-Promoted Asymmetric Alkylation of Aldehydes with Dialkylzincs. J. Am. Chem. Soc. 120, 9800-9809 (1998). 
10. Geiger, Y., Achard, T., Maisse-François, A. \& Bellemin-Laponnaz, S. Observation of Hyperpositive Non-Linear Effects in Asymmetric Catalysis. Nat. Catal. (2020) doi:10.1038/s41929-020-0441-1.

11. Kitamura, M. et al. Enantiomer Recognition of Asymmetric Catalysts. Thermodynamic Properties of Homochiral and Heterochiral Dimers of the Methylzinc Alkoxide Formed from Dimethylzinc and Enantiomeric 3-exo-(Dimethylamino)isoborneol. J. Phys. Chem. 98, 12776-12781 (1994).

12. Sibi, M. \& Liu, M. Reversal of Stereochemistry in Enantioselective Transformations. Can they be Planned or are they Just Accidental? Curr. Org. Chem. 5, 719-755 (2001).

13. Escorihuela, J., Burguete, M. I. \& Luis, S. V. New advances in dual stereocontrol for asymmetric reactions. Chem. Soc. Rev. 42, 5595-5617 (2013).

14. Beletskaya, I. P., Nájera, C. \& Yus, M. Stereodivergent Catalysis. Chem. Rev. 118, 5080-5200 (2018).

15. Cao, W., Feng, X. \& Liu, X. Reversal of enantioselectivity in chiral metal complexcatalyzed asymmetric reactions. Org. Biomol. Chem. 17, 6538-6550 (2019).

16. Porte, A. M., Reibenspies, J. \& Burgess, K. Design and Optimization of New Phosphine Oxazoline Ligands via High-Throughput Catalyst Screening. J. Am. Chem. Soc. 120, 9180-9187 (1998).

17. Du, H., Long, J., Hu, J., Li, X. \& Ding, K. 3,3“-Br2-BINOL-Zn Complex: A Highly Efficient Catalyst for the Enantioselective Hetero-Diels-Alder Reaction. Org. Lett. 4, 4349-4352 (2002).

18. Du, H., Zhang, X., Wang, Z. \& Ding, K. One catalyst for two distinct reactions: sequential asymmetric hetero Diels-Alder reaction and diethylzinc addition. Tetrahedron 61, 94659477 (2005).

19. Schmidt, B. \& Seebach, D. Katalytische und stöchiometrische enantioselektive Additionen von Diethylzink an Aldehyde mit Hilfe eines neuartigen chiralen Spirotitanats. Angew. Chem. 103, 100-101 (1991). 
20. Schmidt, B. \& Seebach, D. 2,2-Dimethyl- $\alpha, \alpha, \alpha^{\prime}, \alpha^{\prime}$-tetra(naphth-2-yl)-1,3-dioxolan-4,5dimethanol (DINOL) für die Titanat-vermittelte, enantioselektive Addition von Diethylzink an Aldehyde. Angew. Chem. 103, 1383-1385 (1991).

21. Seebach, D. et al. On the Mechanisms of Enantioselective Reactions Using $\alpha, \alpha, \alpha^{\prime}, \alpha^{\prime}-$ Tetraaryl-1,3-dioxolane-4,5-dimethanol(TADDOL)-Derived Titanates: Differences between C2- and C1-symmetrical TADDOLs - facts, implications and generalizations. Helv. Chim. Acta 75, 2171-2209 (1992).

22. Danjo, H., Higuchi, M., Yada, M. \& Imamoto, T. P-stereogenic P/N hybrid ligands: a remarkable switch in enantioselectivity in palladium-catalyzed asymmetric allylation. Tetrahedron Lett. 45, 603-606 (2004).

23. Shao, Z., Wang, J., Ding, K. \& Chan, A. S. C. Unprecedented Effects of Additives and Ligand-to-Metal Ratio on the Enantiofacial Selection of Copper-Catalyzed Alkynylation of a-Imino Ester with Arylacetylenes. Adv. Synth. Catal. 349, 2375-2379 (2007).

24. Peng, F., Shao, Z. \& Chan, A. S. C. Copper(I)-catalyzed enantioselective alkynylation of a-imino esters: ligand-to-metal ratio effects and mechanistic studies. Tetrahedron: Asymmetry 21, 465-468 (2010).

25. Burguete, M. I., Collado, M., Escorihuela, J. \& Luis, S. V. Efficient Chirality Switching in the Addition of Diethylzinc to Aldehydes in the Presence of Simple Chiral a-Amino Amides. Angew. Chem. Int. Ed. 46, 9002-9005 (2007).

26. Li, J. et al. Unusual Ligand-to-Metal-Ratio-Controlled Bidirectional Enantioselectivity in Pd-Catalysed [3+3]-Annulation of Morita-Baylis-Hillman Acetate. Eur. J. Org. Chem. 2017, 6961-6965 (2017).

27. Cruz, A., Irene Padilla-Martínez, I. \& Esther Bautista-Ramirez, M. N-Substituted Ephedrines as Chiral Auxiliaries in Enantioselective Alkylation Reactions of Carbonyl Compounds. Curr. Org. Synth. 13, 2-40 (2016).

28. Muchow, C., Vannoorenberghe, Y. \& Buono, G. Use of alkaloids and aminoalcohols in catalytic asymmetric induction:Temperature effect on the addition of diethylzinc to benzaldehyde. Tetrahedron Lett. 28, 6163-6166 (1987). 
29. Chaloner, P. A., Langadianou, E. \& Perera, S. A. R. Enantioselective reaction of diethylzinc with arenecarbaldehydes in the presence of ephedrine derivatives. J. Chem. Soc., Perkin Trans. 1 0, 2731-2735 (1991).

30. Soai, K., Yokohama, S. \& Hayasaka, T. Chiral N,N-Dialkylnorephedrines as Catalysts of the Highly Enantioselective Addition of Dialkylzincs to Aliphatic and Aromatic Aldehydes. The Asymmetric Synthesis of Secondary Aliphatic and Aromatic Alcohols of High Optical Purity. J. Org. Chem. 4264-4268 (1991).

31. Fitzpatrick, K., Hulst, R. \& Kellogg, R. M. Thiol and disulfide derivatives of ephedra alkaloids 2: A mechanistic study of their effect on the addition of diethyl zinc to benzaldehyde. Tetrahedron: Asymmetry 6, 1861-1864 (1995).

32. Potter, G. D., Baird, M. C. \& Cole, S. P. C. A new series of titanocene dichloride derivatives bearing chiral alkylammonium groups; assessment of their cytotoxic properties. Inorg. Chim. Acta 364, 16-22 (2010).

\section{Acknowledgments}

The authors gratefully acknowledge the CNRS and the Ministère de l'Enseignement Supérieur et de la Recherche (MESR) for a PhD grant to Y.G. This work is a part of the Strasbourg IDEX program and we thank the NIE Labex for support (ANR-11-LABX0058_NIE).

\section{Author contributions}

Y.G. performed the synthetic experiments and developed the studied catalytic models, along with their mathematical expressions. A.M.-F. and T.A. participated in data analyses. S.B.-L. conceptualized and supervised the study and wrote the manuscript with Y.G. 